\title{
Sub-Shot-Noise Transmission Measurement Enabled by Active Feed-Forward of Heralded Single Photons
}

\author{
J. Sabines-Chesterking, ${ }^{1}$ R. Whittaker, ${ }^{1}$ S. K. Joshi, ${ }^{2}$ P. M. Birchall, ${ }^{1}$ P. A. Moreau, ${ }^{1}$ A. McMillan, ${ }^{1}$ \\ H. V. Cable, ${ }^{1}$ J. L. O'Brien, ${ }^{1}$ J. G. Rarity, ${ }^{1}$ and J. C. F. Matthews ${ }^{1}$ \\ ${ }^{1}$ Quantum Engineering Technology Labs, H. H. Wills Physics Laboratory and Department of Electrical \\ and Electronic Engineering, University of Bristol, Bristol BS8 1FD, United Kingdom \\ ${ }^{2}$ Institute for Quantum Optics and Quantum Information (IQOQI), Austrian Academy of Sciences, \\ Boltzmanngasse 3, A-1090 Vienna, Austria
}

(Received 7 December 2016; revised manuscript received 26 April 2017; published 17 July 2017; corrected 6 October 2017)

\begin{abstract}
Harnessing the unique properties of quantum mechanics offers the possibility of delivering alternative technologies that can fundamentally outperform their classical counterparts. These technologies deliver advantages only when components operate with performance beyond specific thresholds. For optical quantum metrology, the biggest challenge that impacts on performance thresholds is optical loss. Here, we demonstrate how including an optical delay and an optical switch in a feed-forward configuration with a stable and efficient correlated photon-pair source reduces the detector efficiency required to enable quantum-enhanced sensing down to the detection level of single photons and without postselection. When the switch is active, we observe a factor of improvement in precision of 1.27 for transmission measurement on a per-input-photon basis compared to the performance of a laser emitting an ideal coherent state and measured with the same detection efficiency as our setup. When the switch is inoperative, we observe no quantum advantage.
\end{abstract}

DOI: 10.1103/PhysRevApplied.8.014016

\section{INTRODUCTION}

Quantum mechanics quantifies the highest precision that is achievable in each type of optical measurement [1-3]. Single-photon probes measured with single-photon detectors are, in principle, optimal for gaining the most precision per unit intensity when measuring optical transmission [4]. Entangled multiple-photon states can be engineered to achieve quantum-enhanced precision in interferometry [3] and parameter estimation. However, in practice, optical loss and low component efficiencies prevent an advantage from being achieved using single-photon detectors [5]. One approach is to use postselection to ignore negative measurement outcomes that arise due to loss while recording only positive results. Postselection has proven to be highly successful for observing the underlying physics in proof-of-principle experiments while testing quantum technology [6]. For quantum metrology, however, postselection used on its own leads to more photons being exposed to a measured sample than are used for data analysis, and it therefore generally leads to worse overall performance than using classical strategies [7]. Another way to reduce the impact of lower component efficiency is to incorporate fast optical switching and an optical delay with schemes that are based on the heralded generation of quantum states [8]. Switching enables the use of a quantum state conditioned on the successful detection of a correlated signal-this is referred to as feed-forward and can be used to engineer quantum states that have increasing complexity and utility for quantum metrology $[9,10]$.
Feed-forward is key for demonstrations of optical quantum computing [11], it has been used in experiments that increase the generation rate [12-16] and signal-tonoise ratio [17] of heralded single photons, it has been used to calibrate single-photon detectors [18], and it has also been applied to gather evidence of single-photon sensitivity in animal vision [19]. Jakeman and Rarity proposed in Ref. [8] using feed-forward with correlated photon pairs to enable sub-shot-noise optical transmission measurements when component efficiency is otherwise not sufficient to permit a quantum advantage in passive direct detection [20-22]. However, despite becoming identified as a key to more general multiphoton-entangled quantum-state engineering for quantum metrology $[9,10]$, feed-forward has not been implemented for quantum-enhanced parameter estimation. Here, we implement the proposal featured in Ref. [8] (Fig. 1) to realize sub-shot-noise measurement of transmissivity, using single-photon detectors that are too low in efficiency to enable sub-shot-noise performance in a passive measurement.

\section{THEORY}

The transmissivity $\eta$ of a sample is, in general, estimated by measuring the reduction of light intensity from a known mean input value $\bar{N}_{\text {in }}$ to a reduced mean value $\bar{N}_{\text {out }}$ according to $\eta=\bar{N}_{\text {in }} / \bar{N}_{\text {out }}$. The precision with which $\eta$ can be measured is dependent on the type of light used to probe the channel. When estimating $\eta$ with an ideal 


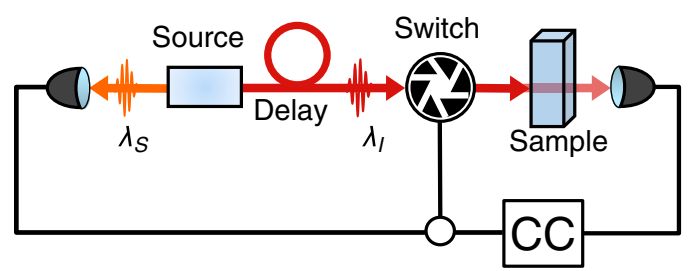

FIG. 1. Photon-pair feed-forward transmission measurement. Photon pairs of signal $\left(\lambda_{S}\right)$ and idler $\left(\lambda_{I}\right)$ photons are simultaneously emitted into two channels. Once a signal photon is detected, it opens a switch in the idler photon's channel to allow probing of a sample with the idler photon. The transmission estimate is obtained from the ratio of the number of coincidence detection (CC) and signal-photon detection events.

coherent-state probe $|\alpha\rangle$, the precision will be given by $\left(1 / \Delta^{2} \eta\right)_{\alpha}=\nu \bar{N}_{\text {in }} / \eta$, where $\bar{N}_{\text {in }}$ is the average number of probe photons and $\nu$ is the number of repetitions of the measurement. This is the shot-noise limit and it is the upper bound on the precision achievable with classical measurements [3]. Higher precision can therefore be achieved by increasing the input intensity and the number of repetitions. For a fixed intensity and a fixed number of repetitions $\nu$, nonclassical states of light can provide an enhancement in precision over coherent-state probes. The photon-number probability distribution of a Fock state of $\bar{N}_{\text {in }}=N_{\text {in }}$ photons after passing through a lossy channel follows the binomial distribution $P\left(N_{\text {out }}, N_{\text {in }}, \eta\right)=$

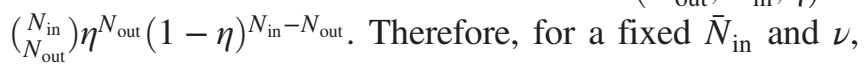
the Fock-state probe achieves a higher precision than the coherent state [4]:

$$
\left(1 / \Delta^{2} \eta\right)_{F}=\nu \bar{N}_{\text {in }} / \eta(1-\eta)>\left(1 / \Delta^{2} \eta\right)_{\alpha}
$$

The performance of Fock states can be accessed by using correlated photon pairs generated from a spontaneous parametric down-conversion (SPDC) process. Signal photons of each correlated pair are sent directly for detection to herald the presence of the corresponding idler photon which is used to probe a sample. The transmissivity of the photons through the sample can then be estimated from the Klyshko (heralding) efficiency [23] of the idler channel $\eta_{I}$, which is the ratio of the number of photons detected coincidentally across the two channels $N_{C}$ and the total number of detected herald (signal) photons $N_{S}: \eta_{I}=\bar{N}_{C} / \bar{N}_{S}$.

To obtain a quantum advantage using the Klyshko efficiency as the transmission estimator, it is required to have a strong correlation between the number of signal and idler photons such that the difference between the coincidence and the signal count is due only to the absorption of the sample [20-22]. This is generally not the case when there is loss in either the signal or the idler channel, so when system performance prohibits having a high correlation, one can selectively analyze subsets of recorded data in postselection to observe sub-shot-noise behavior [24]. However, in practice, a sample measured with postselected events will be overexposed, with photons that are unaccounted for due to lost counterpart heralding photons. Postselection then results in a strategy that performs worse than using a coherent state when analysis is normalized to per input probe photon. By introducing an optical switch into the setup, as sketched in Fig. 1, that allows only photons incident on a sample when a signal photon has been successfully detected, we increase the level of correlation and suppress the detrimental effect of loss in the signal channel. Doing so changes the efficiency parameter $\eta_{S}=N_{C} / N_{I}$ from being the transmissivity of the signal channel into a parameter that describes the percentage of the idler photons detected after the switch that are correlated to detected signal photons. The parameter $1-\eta_{S}$ therefore describes the leakage of unheralded idler photons through the switch. However, there are still three main mechanisms that can degrade the performance of the photon-pair strategy using a switch. The first is loss of the idler photon in the photon source (including feedforward optics) $1-\eta_{\text {source }}$ and at the detector $1-\eta_{\text {det }}$, which together with sample transmission $\eta$ redefines the Klyshko efficiency $\eta_{I}=\eta_{\text {source }} \eta \eta_{\text {det }}$. For a single-photon Fock state, $\rho=|1\rangle\langle 1|, \eta_{I}$ modifies the state according to

$$
\rho \rightarrow \rho^{\prime}=\left(1-\eta_{I}\right)|0\rangle\left\langle 0\left|+\eta_{I}\right| 1\right\rangle\langle 1|,
$$

which still follows a (sub-Poissonian) binomial distribution and therefore still outperforms coherent states per input photon. As loss increases $\eta_{\text {source }} \eta_{\text {det }} \rightarrow 0$, however, the measured photon-number distribution tends towards Poissonian. The second degradation mechanism is imperfect optical switching that allows unheralded photons to leak through the sample; this is quantified by $1-\eta_{S}$. The third mechanism is dark counts in the signal detector that can herald false idler photons into the idler channel. The effect of dark counts becomes negligible when dark-count rates are low compared to signal-photon detection rates. We plot examples of the effect of loss and leakage in Fig. 2, in terms of the ratio between the precision achievable using a Fock state that has either been degraded by loss or incorrectly heralded with switch leakage, denoted $1 /\left(\Delta^{2} \eta\right)_{F^{\prime}}=\eta_{S} / \eta\left(1-\eta \eta_{I}\right)$, and the precision achievable with a coherent-state probe $1 /\left(\Delta^{2} \eta\right)_{\alpha}=1 / \eta$ with the same detector efficiency. This ratio is a figure of merit that determines when a quantum advantage is obtained - that is, when $R=\eta_{S} /\left(1-\eta \eta_{I}\right)>1$. Note that this expression leads to the condition found in Ref. [8], where it was shown that, for obtaining a quantum advantage over using a coherent state, it is necessary that

$$
\eta_{I}+\eta_{S}>1
$$




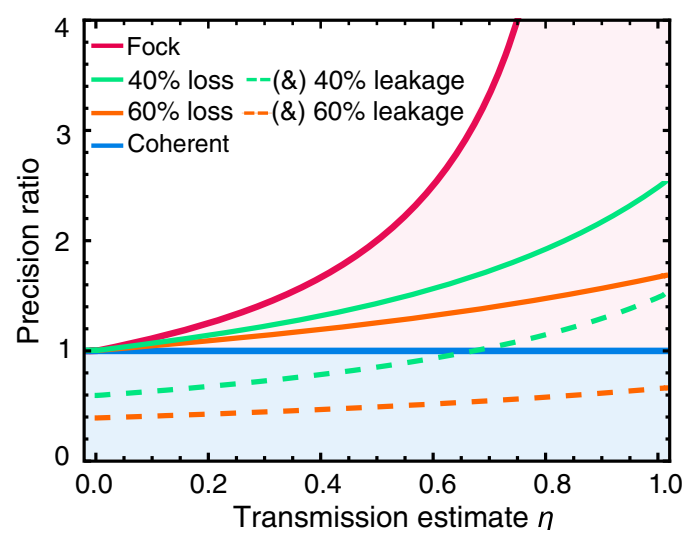

FIG. 2. Theoretical performance of the photon-pair feedforward transmission measurement. Precision-achievable relative to coherent states is plotted as a function of sample transmission $\eta$ for an average input intensity of $\bar{N}=1$ photons. The pink curve represents the ideal case of a heralded Fock state with no setup loss $\left(\eta_{\text {det }} \eta_{\text {source }}=1\right)$, aside from the sample's transmission, and no switch leakage $\left(\eta_{S}=1\right)$. Illustrating the effect of experimental imperfections, the green and orange solid curves correspond to mixed states with setup losses $\eta_{\text {det }} \eta_{\text {source }}=0.4$ and $\eta_{\text {det }} \eta_{\text {source }}=$ 0.6 and with $\eta_{S}=1$. The green and orange dashed curves represent, respectively, performance with $\eta_{\operatorname{det}} \eta_{\text {source }}=0.4$ and leakage $1-\eta_{S}=0.4$, and $\eta_{\text {det }} \eta_{\text {source }}=0.6$ and $1-\eta_{S}=0.6$. The blue curve represents the shot-noise limit. The light-pink region reflects the area where there is a quantum advantage.

\section{EXPERIMENTAL SETUP}

The experimental setup we use to implement the feedforward transmission measurement is shown in Fig. 3. Photon pairs are generated via collinear type-II SPDC using a periodically poled potassium titanyl phosphate crystal (PPKTP), pumped with a continuous-wave (cw) laser diode $\left(\lambda_{p}=403.9 \mathrm{~nm}\right)$ and spectrally tuned by controlling its temperature. The wavelengths of the signal and idler photons are $\lambda_{s}=792 \mathrm{~nm}$ and $\lambda_{i}=824 \mathrm{~nm}$, each with a spectral width of $\pm 0.4 \mathrm{~nm}$. After down-conversion, the pump is removed using a 715-nm long-pass filter (LPF) and a 50-nm-wide bandpass filter (BPF) centered at $808 \mathrm{~nm}$. Photon pairs are split deterministically using a polarization beam splitter (PBS), sending the idler photon through the delay line while the correlated signal photon is collected with a single-mode fiber and detected using an avalanche photodiode (APD).

The detected signal photon triggers an optical switch implemented with a Pockels-cell modulator composed of two lithium niobate crystals that rotate the polarization of an incoming photon by $90^{\circ}$ when inactive and preserve the photon's polarization when activated with $200 \mathrm{~V}$. The rise time of this switch is $500 \mathrm{~ns}$. To compensate for any polarization rotations of the delay fiber, the optical modulator is set inside a Sagnac loop, similar to the one reported in Ref. [25], to enable bidirectional operation independent of the input polarization. This strategy is chosen to avoid the higher loss associated with polarization-maintaining fiber and the need for active polarization stabilization. After switching, the idler photon is incident upon a variabletransmission element comprising a half wave plate (HWP) and a PBS to mimic a variable-transmission sample. Since the polarization of the idler photon is mixed after the optical-fiber delay, both the horizontal and vertical polarization components of the idler photon need to experience the same value of $\eta$-we obtain the same polarization by using a calcite beam displacer (BD) and a half wave plate to convert the two polarization components into two path modes with the same polarization. The loss introduced by these two components is $1.4 \%$ and is included in the overall system loss. Both modes then pass through the transmission element and are subsequently focused together

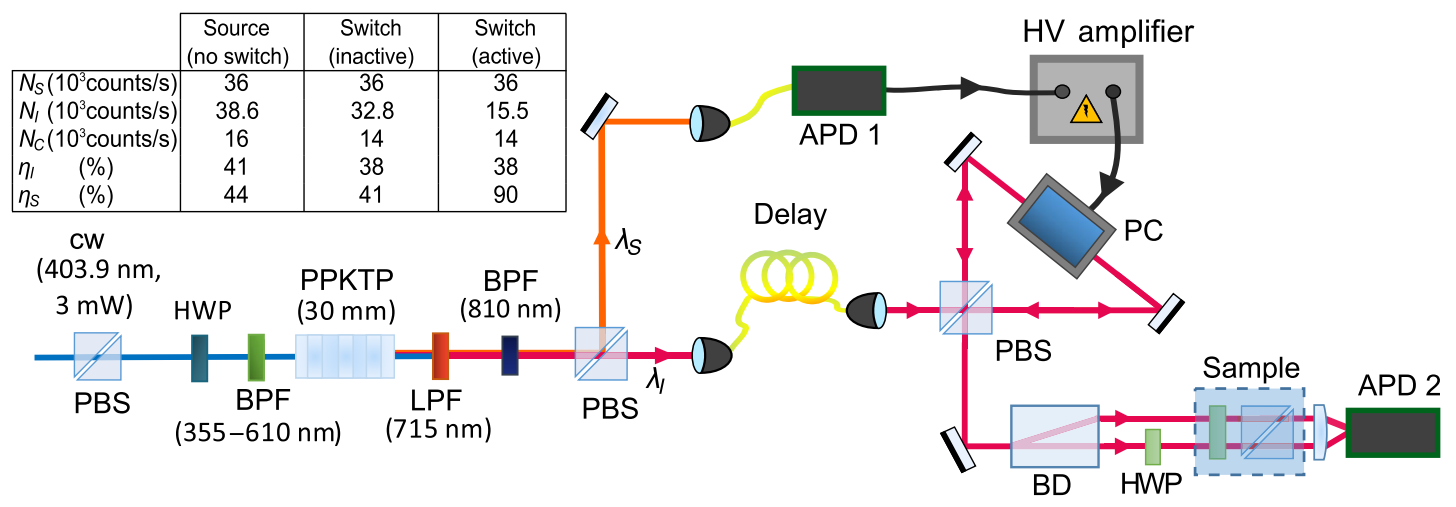

FIG. 3. Experimental setup. Photon pairs are generated via type-II collinear SPDC using a 30 mm PPKTP crystal pumped with a continuous-wave (cw) 404-nm laser. While the idler photon goes through a delay line, the signal photon is detected and triggers an optical switch. The switch is a commercially available free-space Pockels-cell (PC) modulator consisting of two lithium niobate crystals inside a Sagnac loop powered by a high voltage (HV) amplifier. The source is mounted in a cage system to reduce vibrational noise. (Inset table) The approximate mean photon-count rates $\left(N_{S}, N_{I}, N_{C}\right)$ and the efficiencies $\left(\eta_{I}=N_{C} / N_{S}\right.$ and $\left.\eta_{S}=N_{C} / N_{I}\right)$ for the experiment in the configurations of (i) the photon source only, (ii) addition of the switch optical components but left inactive, and (iii) addition of the switch components when activated. 
onto a free-space APD for detection. Before any measurements of transmission, we first characterize the performance of the setup. The efficiencies of the source without the switch are $\eta_{S}=41 \%$ and $\eta_{I}=44 \%$ for the signal and idler channels, respectively, corrected for dark counts but including $\eta_{\text {source }}$ and $\eta_{\text {det }} \sim 65 \%$. After introducing the switch, the efficiency of the idler photon's path (without a sample) is reduced to $\eta_{\text {source }} \eta_{\operatorname{det}}=38 \%$, which means that there is a loss of approximately $15 \%$ in the Sagnac loop and the delay line. The Klyshko efficiency of the signal channel, $\eta_{S}$, increases to approximately $90 \%$, which is less than the ideal $\eta_{S}=100 \%$ due to the approximately $1-\mu \mathrm{s}$ width of the switching window that permits unheralded photons to be leaked through the switch. The pump power is adjusted to minimize this effect, having a detection rate in the signal path of about $36 \times 10^{3}$ counts/s, approximately $15.5 \times 10^{3}$ counts/s in the idler channel, and a coincidence rate of about $14 \times 10^{3}$ counts/s. By comparison, dark-count rates in the signal detector are low (about $0.6 \times 10^{3}$ counts $/ \mathrm{s}$ ) and are accounted for when estimating $\eta_{I}$ by subtracting their average number (characterized in the setup with signal- and idler-photon beams blocked) from the total number of detected counts recorded in our measurement. This means that the contribution from dark counts which can lead to false heralding events is about $1.5 \%$.

To verify that the source is heralding true single photons, we measure the second-order correlation function of the idler mode using the triple-coincidence method reported in Ref. [26], obtaining a value of $g^{(2)}(0)=0.031 \pm 0.002$ [where $g^{(2)}(0)=0$ corresponds to perfect single photons and $g^{(2)}(0)=1$ corresponds to Poisson-distributed light]. We estimate the transmission of the sample $\eta$ as the ratio between $\eta_{I}$ measured at different sample transmission conditions and $\eta_{\text {source }} \eta_{\text {det }}$, which we characterize by measuring $\eta_{I}$ with the sample transmission set to $\eta=1$. The statistical precision of the transmission estimate per probe input to the sample is given by the inverse of

$$
\Delta^{2} \eta=\operatorname{Var}\left(\frac{\eta_{\mathrm{I}}}{\eta_{\text {source }}}\right) \bar{N}_{\text {probe }}
$$

where $\bar{N}_{\text {probe }}$ is the average number of probe photons given by the number of detected idler photons $\left(N_{I}\right)$ corrected for the percentage of idler photons that are successfully heralded $\left(\eta_{S}\right)$, absorbed photons by the sample $(\eta)$, average number of dark counts of the detector $\left(\bar{N}_{D}\right)$, and detector efficiency $\left(\eta_{\text {det }}\right)$ :

$$
\bar{N}_{\text {probe }}=\frac{\bar{N}_{I}}{\eta_{\text {det }} \eta \eta_{S}}-\bar{N}_{D}
$$

\section{RESULTS}

In Fig. 4, we present the precision achievable with our feed-forward transmission measurement setup, with respect
- Switch active - Classical limit $\Delta$ Switch inactive

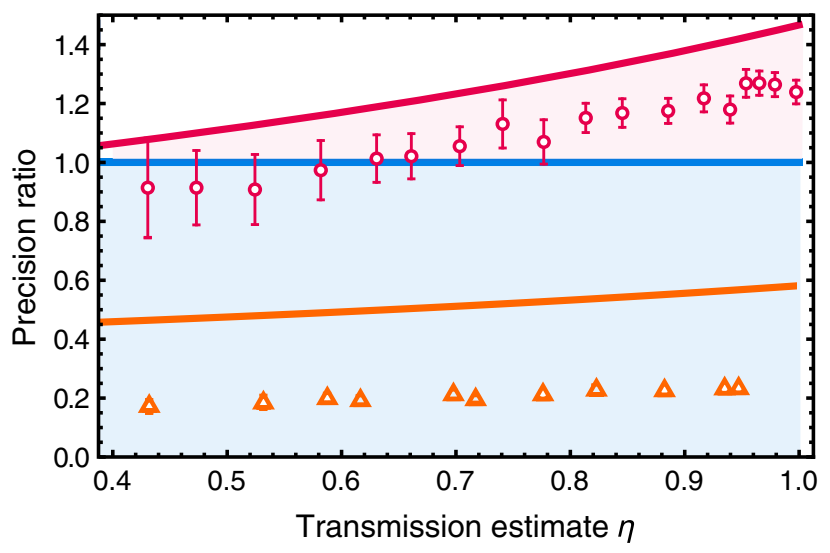

FIG. 4. Experimental results. The pink circles correspond to the estimated experimental advantage compared to a coherent state having the same detector efficiency. Each point corresponds to 3000 repetitions of measurements taken with an integration time of $0.2 \mathrm{~s}$ and a coincidence window of $30 \mathrm{~ns}$. The orange triangles correspond to the performance of the scheme when the switch is not active (error bars are too small to be seen). The pink solid line corresponds to the expected trend for mixed states with setup efficiency $\eta_{\text {source }} \eta_{\text {det }}=38 \%$ and $1-\eta_{S}=10 \%$ leakage. The orange line corresponds to a mixed state with the same setup efficiency but with $1-\eta_{S}=62 \%$ leaked or unheralded photons. The discrepancy between the solid lines and the experimental points is attributed to various sources of noise, including vibrational motion of the fiber couplers, temperature fluctuations of the crystal, and electrical noise from detectors. Error bars are obtained by calculating the variance of binned sets of data points.

to the theoretical precision achievable with a coherent-state scheme using the same detector efficiency [27]. We make this comparison by computing the ratio of precision of the two schemes, as in Fig. 2, and we observe a factor of improvement of up to $1.27 \pm 0.08$ for $\eta=0.97$, and a quantum advantage is observable down to a sample transmission of $\eta=0.65$. When we turn off the optical switch, the performance of the setup is far below that of the coherent-state strategy.

\section{CONCLUSION}

Using feed-forward for measurement is advantageous when it is desired to probe an object with a controlled number of photons [19,28,29]. Solid-state sources of photons, such as quantum dots, could also be used for such purposes. They can operate with megahertz emission rates [30], they can be used with high heralding efficiency [31], and they can emit higher energy photons [32] than those demonstrated in this paper-however, the higher specification solid-state photon sources currently require additional resources, particularly cryogenic cooling and narrow-band filtering from photonic-structure engineering, that can limit practicality and add cost to development. Practical application of using feed-forward with 
spontaneous sources for measurement will be aided by improvements in the brightness of the source [10] and the switching speed. Increasing the precision obtainable per unit intensity will come with improvements in the loss budget of the setup and increasing detector efficiency. State-of-the-art SPDC sources using superconducting detectors have reported Klyshko efficiencies of 83\% [33]; such an efficiency would already translate into about a fivefold advantage in precision in our setup. Incorporating the wavelength tunability available in SPDC sources can enable a sub-shot-noise measurement of spectral response [24]. The polarization-independent switch used in our experiment could also be useful as the feed-forward mechanism to engineer quantum states that are more complex and have more utility than single photons $[9,10]$.

The data in this article are available online [34].

\section{ACKNOWLEDGMENTS}

We thank M. Loutit and A. Neville for their technical assistance. This work was supported by Engineering and Physical Sciences Research Council (EPSRC), European Research Council (ERC), and UK Quantum Technology Hub in Quantum Enhanced Imaging (QuantIC). J. C. F. M. and J.G.R. acknowledge fellowship support from the Engineering and Physical Sciences Research Council. J. C. F. M. and J. G. R. acknowledge fellowship support from the Engineering and Physical Sciences Research Council under Grant Code No. EP/M024385/1.

[1] Crispin Gardiner and Peter Zoller, Quantum Noise: A Handbook of Markovian and Non-Markovian Quantum Stochastic Methods with Applications to Quantum Optics, Springer Series in Synergetics Vol. 56 (Springer Science+Business Media, New York, 2004).

[2] Roman Schnabel, Nergis Mavalvala, David E. McClelland, and Ping K. Lam, Quantum metrology for gravitational wave astronomy, Nat. Commun. 1, 121 (2010).

[3] V. Giovannetti, S. Lloyd, and L. Maccone, Advances in quantum metrology, Nat. Photonics 5, 222 (2011).

[4] G. Adesso, F. Dell'Anno, S. De Siena, F. Illuminati, and L. A. M. Souza, Phys. Rev. A 79, 040305(R) (2009).

[5] Nicholas Thomas-Peter, Brian J. Smith, Animesh Datta, Lijian Zhang, Uwe Dorner, and Ian A. Walmsley, RealWorld Quantum Sensors: Evaluating Resources for Precision Measurement, Phys. Rev. Lett. 107, 113603 (2011).

[6] J. L. O'Brien, A. Furusawa, and J. Vuckovic, Photonic quantum technologies, Nat. Photonics 3, 687 (2009).

[7] N. Thomas-Peter, B. J. Smith, A. Datta, L. Zhang, U. Dorner, and I. A. Walmsley, Real-World Quantum Sensors: Evaluating Resources for Precision Measurement, Phys. Rev. Lett. 107, 113603 (2011).

[8] E. Jakeman and J. G. Rarity, The use of pair production processes to reduce quantum noise in transmission measurements, Opt. Commun. 59, 219 (1986).
[9] H. Cable and J. P. Dowling, Efficient Generation of Large Number-Path Entanglement Using Only Linear Optics and Feed-Forward, Phys. Rev. Lett. 99, 163604 (2007).

[10] Kevin T. McCusker and Paul G. Kwiat, Efficient Optical Quantum State Engineering, Phys. Rev. Lett. 103, 163602 (2009).

[11] Robert Prevedel, Philip Walther, Felix Tiefenbacher, Pascal Böhi, Rainer Kaltenbaek, Thomas Jennewein, and Anton Zeilinger, High-speed linear optics quantum computing using active feed-forward, Nature (London) 445, 65 (2007).

[12] Xiao-song Ma, Stefan Zotter, Johannes Kofler, Thomas Jennewein, and Anton Zeilinger, Experimental generation of single photons via active multiplexing, Phys. Rev. A 83, 043814 (2011).

[13] Matthew J. Collins, Chunle Xiong, Isabella H. Rey, Trung D. Vo, Jiakun He, Shayan Shahnia, Christopher Reardon, Thomas F. Krauss, M. J. Steel, Alex S. Clark et al., Integrated spatial multiplexing of heralded single-photon sources, Nat. Commun. 4, 2582 (2013).

[14] F. Kanadea, B. G. Christensen, J. J. Wong, H. S. Park, K. T. McCusker, and P. G. Kwiat, Time-multiplexed heralded single-photon source, Optica 2, 1010 (2015).

[15] Gabriel J. Mendoza, Raffaele Santagati, Jack Munns, Elizabeth Hemsley, Mateusz Piekarek, Enrique MartínLópez, Graham D. Marshall, Damien Bonneau, Mark G. Thompson, and Jeremy L. O'Brien, Active temporal and spatial multiplexing of photons, Optica 3, 127 (2016).

[16] R. J. A. Francis-Jones, R. A. Hoggarth, and P. J. Mosley, All-fibre multiplexed source of high-purity heralded single photons, Optica 3, 1270 (2017).

[17] G. Brida, I. P. Degiovanni, M. Genovese, F. Piacentini, P. Traina, A. Della Frera, A. Tosi, A. Bahgat Shehata, C. Scarcella, A Gulinatti et al., An extremely low-noise heralded single-photon source: A breakthrough for quantum technologies, Appl. Phys. Lett. 101, 221112 (2012).

[18] G. Brida, M. Genovese, and M. Gramegna, Twin-photon techniques for photo-detector calibration, Laser Phys. Lett. 3, 115 (2006).

[19] Nam Mai Phan, Mei Fun Cheng, Dmitri A. Bessarab, and Leonid A. Krivitsky, Interaction of Fixed Number of Photons with Retinal Rod Cells, Phys. Rev. Lett. 112, 213601 (2014).

[20] A. Heidmann, R. J. Horowicz, S. Reynaud, E. Giacobino, C. Fabre, and G. Camy, Observation of Quantum Noise Reduction on Twin Laser Beams, Phys. Rev. Lett. 59, 2555 (1987).

[21] J. G. Rarity, P. R. Tapster, and E. Jakeman, Observation of sub-Poissonian light in parametric downconversion, Opt. Commun. 62, 201 (1987).

[22] G. Brida, M. Genovese, and I. Rou Berchera, Experimental realization of sub-shot-noise quantum imaging, Nat. Photonics 4, 227 (2010).

[23] D. N. Klyshko, Use of two-photon light for absolute calibration of photoelectric detectors, Sov. J. Quantum Electron. 10, 1112 (1980).

[24] Rebecca Whittaker, Chris Erven, Alex Neville, Monica Berry, J. L. O'Brien, Hugo Cable, and J. C. F. Matthews, Absorption spectroscopy at the ultimate quantum limit from single-photon states, New J. Phys. 19, 023013 (2017). 
[25] Tien Tjuen Ng, Darwin Gosal, Antía Lamas-Linares, and Christian Kurtsiefer, Sagnac-loop phase shifter with polarization-independent operation, Rev. Sci. Instrum. 82, 013106 (2011).

[26] M. Beck, Comparing measurements of $g^{(2)}(0)$ performed with different coincidence detection techniques, J. Opt. Soc. Am. B 24, 2972 (2007).

[27] P.-A. Moreau, J. Sabines-Chesterking, R. Whittaker, S. K. Joshi, A. McMillan, J. G. Rarity, and J. C. F. Matthews, Demonstrating an absolute quantum advantage in direct absorption measurement, arXiv:1611.07871.

[28] F. Wolfgramm, C. Vitelli, F. A. Beduini, N. Godbout, and M. W. Mitchell, Entanglement-enhanced probing of a delicate material system, Nat. Photonics 7, 28 (2013).

[29] Jonathan N. Tinsley, Maxim I. Molodtsov, Robert Prevedel, David Wartmann, Jofre Espigulé-Pons, Mattias Lauwers, and Alipasha Vaziri, Direct detection of a single photon by humans, Nat. Commun. 7, 12172 (2016).

[30] Thang B. Hoang, Gleb M. Akselrod, and Maiken H. Mikkelsen, Ultrafast room-temperature single photon emission from quantum dots coupled to plasmonic nanocavities, Nano Lett. 16, 270 (2016).

[31] Xing Ding, Yu He, Z.-C. Duan, Niels Gregersen, M.-C. Chen, S. Unsleber, Sebastian Maier, Christian Schneider, Martin Kamp, Sven Höfling et al., On-Demand Single Photons with High Extraction Efficiency and Near-Unity Indistinguishability from a Resonantly Driven Quantum Dot in a Micropillar, Phys. Rev. Lett. 116, 020401 (2016).

[32] Je-Hyung Kim, Young-Ho Ko, Su-Hyun Gong, Suk-Min Ko, and Yong-Hoon Cho, Ultrafast single photon emitting quantum photonic structures based on a nano-obelisk, Sci. Rep. 3, 2150 (2013).

[33] Sven Ramelow, Alexandra Mech, Marissa Giustina, Simon Gröblacher, Witlef Wieczorek, Jörn Beyer, Adriana Lita, Brice Calkins, Thomas Gerrits, Sae Woo Nam et al., Highly efficient heralding of entangled single photons, Opt. Express 21, 6707 (2013).

[34] DOI: 10.5523/bris.9lm4e9t18ffm26ri0cbe7yr4p. 